\title{
Desinfección de Alicates de Ortodoncia. Actualización de Recomendaciones en Contexto de COVID- 19. Revisión de la Literatura
}

\author{
Disinfection of Orthodontic Pliers. Recommendations Update \\ in the Context of COVID-19. Literature Review
}

\begin{abstract}
María Soledad Munizaga Naveillan'; Eduardo Antonio Alvarez Palacios²; Germán Hempel Souper²; María Ignacia Sat Yaber²; Francisca Carranza Morales² \& Katerín Terán Quezada²
\end{abstract}

MUNIZAGA, N. M. S.; ALVAREZ, P. E. A.; HEMPEL, S. G.; SAT, Y. M. I.; CARRANZA, M. F. \& TERÁN, Q. K. Desinfección de alicates de ortodoncia. Actualización de recomendaciones en contexto de COVID- 19. Revisión de la Literatura. Int. J. Odontostomat., 15(3):602-609, 2021.

RESUMEN: El adecuado conocimiento de definiciones, normas, procedimientos, uso de antisépticos y desinfectantes, permite contar con las herramientas para evitar la diseminación de agentes infecciosos y proporcionar el conocimiento para el uso racional de estos. El objetivo de este trabajo es presentar de manera estructurada y ordenada la información disponible respecto de la desinfección de alicates de ortodoncia y proporcionar una guía para el control óptimo de infecciones. Se realizó una búsqueda mediante PubMed, MEDLINE y Google Scholar, sin importar idioma, desde el año 2000 a abril 2021. Se establecieron los desinfectantes más recomendados para la desinfección de alicates de ortodoncia y las consideraciones en su uso. Además, se determinaron los riesgos de corrosión y recomendaciones para evitarlas. Es importante contar con información actualizada que se pueda utilizar para desarrollar un programa óptimo de asepsia, sobre todo en el contexto actual de pandemía.

PALABRAS CLAVE: COVID, COVID-19, COVID-2019, 2019-nCoV, SARS-CoV-2, coronavirus, desinfection pliers, orthodontic pliers, desinfección, alicates de ortodoncia.

\section{INTRODUCCIÓN}

El interés en desarrollar métodos para controlar la infección de enfermedades no es nuevo. Desde la década de los 70 , con el aumento significativo de casos de hepatitis B y Síndrome de Inmunodeficiencia Adquirida, se reconoció la importancia de la investigación científica y la rigurosidad en los estándares de control de infecciones (Venturelli et al., 2009; Almeida et al., 2012). Actualmente las infecciones asociadas a la atención en salud (IAAS) continúan siendo un problema trascendental, tanto en la atención pública como privada, y el COVID-19 nos plantea un desafío en la revisión y endurecimiento de las medidas sanitarias.

La práctica odontológica, y en particular la ortodoncia, se caracteriza por la alta rotación de pa- cientes y la variedad de vehículos para la transmisión de enfermedades infecciosas (Ghanbarzadeh et al., 2014; Jain \& Patel, 2020; Kaur et al., 2020). Según la Asociación Dental Americana (ADA), se estimó que los profesionales dentales y los pacientes pueden verse afectados por alrededor de 40 tipos diferentes de enfermedades infecciosas solo en los procedimientos clínicos de rutina. Todas las enfermedades infecciosas se inician a partir de la exposición del cuerpo a microorganismos patógenos. En la cavidad oral se encuentran más microorganismos que en cualquier otra parte del cuerpo. Según un estudio, los ortodoncistas tienen la segunda incidencia más alta de Hepatitis B entre los Odontólogos. Para la protección tanto del médico como del paciente, el control de

\footnotetext{
1 Facultad de Odontología, Universidad de los Andes, Santiago, Chile.

2 Postítulo de Ortodoncia y Ortopedia DMF, Facultad de Odontología, Universidad de Chile, Santiago, Chile.
} 
infecciones es de suma importancia para prevenir la propagación de enfermedades infecciosas (Almeida et al.; Kalra et al., 2015; Çelikel et al., 2018; Jankare et al., 2019; Gurzawska-Comis et al., 2020; Turkistani, 2020).

El adecuado conocimiento de definiciones, normas, procedimientos, uso de antisépticos y desinfectantes, permite al profesional de la salud contar con las herramientas para evitar la diseminación de agentes infecciosos y proporcionar el conocimiento para el uso racional de estos. Es necesario un adecuado manejo de áreas, un controlado manejo de instrumental crítico, semicritico y no crítico, además de la prevención de infecciones cruzadas por la exposición permanente a fluidos corporales, mucosas y dientes. El instrumental de ortodoncia constituye un factor de riesgo en el equipo odontológico y para los pacientes, por la exposición a diversos agentes patógenos como virus, bacterias y hongos, que se transmiten a través de la sangre y secreciones orales y respiratorias, principalmente aquellos afines al tracto respiratorio superior (Ayyed et al., 2020; Kondody et al., 2021).

Varios estudios han informado la transmisión cruzada de COVID-19 entre los trabajadores de la salud. Este hecho hace que las estrictas medidas de control de infecciones sean un área de preocupación. El período de incubación de esta enfermedad es de 14 hasta 24 días, siendo altamente contagiosa durante este período de latencia, esto nos alerta del peligro potencial en el tratamiento de pacientes asintomáticos y la propagación de infecciones dentro de la clínica de ortodoncia. Además, la generación de aerosoles, una ocurrencia rutinaria en la clínica, es una ruta confirmada de transmisión de infecciones (Cabrera-Tasayco et al., 2020; GurzawskaComis et al.; Suri et al., 2020).

Dada la necesidad de mejorar el control de infecciones, aumentar la conciencia y controlar la contaminación cruzada entre ortodoncistas, asistentes, personal de oficina y pacientes durante la crisis de COVID-19 es esencial evaluar la efectividad real de los métodos comúnmente utilizados por los ortodoncistas para la desinfección de alicates en su práctica diaria.

El objetivo de esta revisión es presentar de manera estructurada y ordenada toda la información disponible respecto a la desinfección de alicates, y proporcionar una guía en nuestra práctica clínica para un control óptimo de infecciones. Se discutirán además las consideraciones clínicas más relevantes.

\section{MATERIAL Y MÉTODO}

Teniendo en cuenta la reciente aparición de COVID-19, la poca información disponible del tema después de iniciada la pandemia y con el fin de realizar una actualización que permita su aplicación en el contexto actual, se considero incluir artículos relacionados a la desinfección de alicates de ortodoncia previo a la pandemia, artículos durante la pandemia y relacionados al COVID. Los estudios se recopilaron de las siguientes bases de datos: PubMed, MEDLINE y Google Scholar. Se realizó una búsqueda comprendida desde el año 2000 hasta abril 2021 utilizando los siguientes términos: COVID, COVID-19, COVID-2019, 2019-nCoV, SARS-CoV2 , coronavirus, desinfection pliers, orthodontic pliers, desinfección, alicates de ortodoncia. Se seleccionaron los estudios que incluían cualquiera de los términos de búsqueda, incluyendo publicaciones en inglés y otros idiomas. Se tradujeron los títulos y resúmenes que no estaban en inglés y se realizó un cribado eliminando los estudios que no tuviesen relación al tema. No se consideraron artículos en los cuales no se pudo obtener el texto completo. Finalmente se obtuvieron 25 artículos que caen dentro del alcance de esta revisión. Además se incorporó la norma №199 vigente del Ministerio de Salud sobre esterilización y desinfección de alto nivel y el manual de desinfección de alicates del Hospital Luis Calvo Mackenna, obtenidos a través de búsqueda manual.

\section{Terminología}

- Esterilización: proceso mediante el cual un artículo, medio o superficie está libre de todos los microorganismos patógenos, ya sea en forma vegetativa o de esporas (Kalra et al.; Jankare et al.). - Desinfección: proceso de destruir o inhibir la mayoría de los microorganismos patógenos e inactivar algunos virus, reduciendo así la contaminación microbiana al nivel de seguridad (Kalra et al.; Jankare et al.).

- Antisepsia: aplicación de productos químicos en tejidos vivos para evitar infecciones (Jankare et al.).

- Asepsia: ambiente libre de gérmenes que implica la destrucción de todos los microorganismos que forman enfermedades en el ambiente de trabajo (Jankare et al.).

- Descontaminación: reducir la fuente microbiana en número para la protección contra la contaminación inesperada y la infección (Jankare et al.). 
MUNIZAGA, N. M. S.; ALVAREZ, P. E. A.; HEMPEL, S. G.; SAT, Y. M. I.; CARRANZA, M. F. \& TERÁN, Q. K. Desinfección de alicates de ortodoncia. Actualización de recomendaciones en contexto de COVID- 19. Revisión de la Literatura. Int. J. Odontostomat., 15(3):602-609, 2021.

\section{Principales objetivos en el control clínico de in- fecciones}

- Reducir el riesgo de infecciones disminuyendo el nivel de exposición a patógenos (Kalra et al.; Jankare et al.).

- Mantener de forma estricta una correcta técnica aséptica (Kalra et al.; Jankare et al.).

- Usar precauciones universales con cada paciente (tratar a cada paciente e instrumento como potencialmente infeccioso) (Kalra et al.; Jankare et al.; Kondody et al.).

- Proteger a los pacientes y al personal de infecciones ocupacionales (Ayyed et al.; Gurzawska-Comis et al.; Kalra et al.; Jankare et al.; Turkistani).

\section{Riesgo de transmisión de enfermedades dentro de la práctica de ortodoncia}

La atención en ortodoncia, al igual que en cualquier especialidad odontológica, requiere proximidad a los pacientes. Desafortunadamente, esto hace que exista un alto riesgo de contraer enfermedades infecciosas (Jain \& Patel). Las recomendaciones actuales para COVID 19 son evitar el contacto de persona a persona y mantener una distancia de 1-2 m entre las personas (García-Camba et al., 2020). Esta recomendación es muy dificil de optimizar en la clínica de ortodoncia poniendo en una situación alta de riesgo al ortodoncista y al asistente dental.

El período de incubación de la infección por Covid- 19 podría llegar hasta los 24 días, generalmente varía de 1 a 14 días, con un promedio de 3 a 7 días, sin dejar de ser contagioso durante este período de latencia (Cabrera-Tasayco et al.; Suri et al.; Kondody et al.). Esto aumenta el riesgo para los ortodoncistas que tienden a ver diariamente un gran volumen de pacientes en un período corto de tiempo de atención. Además, la presencia de portadores asintomáticos, incluidos niños (que componen un alto porcentaje de los pacientes de ortodoncia), eleva aún más el riesgo en el equipo de ortodoncia (Guo et al., 2020; Suri et al.).

Se sabe que las enfermedades infecciosas constituyen un riesgo en la práctica clínica de salud dental, lo que determina la importancia de tomar medidas estrictas para garantizar la seguridad y evitar una posible transmisión dentro de la práctica de ortodoncia. Estudios anteriores han informado de transmisión cruzada de infecciones dentro de clínica odontológica incluidas la hepatitis B y la hepatitis C.
En Estados Unidos, los ortodoncistas ocupan el segundo lugar en la incidencia más alta de contraer hepatitis $B$.

\section{Posibles fuentes de contaminación}

1. Saliva del paciente: Se han informado en varios estudios altas cargas de SARS-CoV-2 en la saliva de pacientes infectados. Además, informaron un gran número de ACE2 (el receptor del SARS-CoV-2) en la lengua humana y la mucosa oral. La cavidad oral es, por tanto, una incubadora y un anfitrión de transmisión de enfermedades (Khatri et al., 2017; Carter, 2020; Grover et al., 2020; Guo et al.; Suri et al.; Turkistani).

2. Aerosol: La generación de aerosoles en la práctica clínica genera un riesgo ya que este podría estar contaminado con la sangre del paciente, la saliva o concentraciones altas de microbios infecciosos que superen las producidas al toser o estornudar. Los aerosoles que contienen gérmenes de 0.5-1.0 mm o menos pueden permanecer en el aire por más tiempo, aumentando el riesgo de ser inhalados y entrar en áreas más profundas del pulmón, lo que representa un riesgo potencial de infección. Esto presenta colectivamente una amenaza alarmante con el COVID-19 altamente contagioso (Ayyed et al.; Carter; Grover et al.; Guo et al.; Khatri et al.; Suri etal.; Turkistani).

3. Suministros e instrumentos de ortodoncia: Los instrumentos de ortodoncia que entran en contacto directo con la saliva y la sangre de los pacientes. Además, la reutilización de bandas de ortodoncia, brackets de ortodoncia, cadenas elastoméricas, fresas de carburo de tungsteno, minitornillos, marcadores de ortodoncia y retractores fotográficos, etc, sin esterilización y desinfección adecuadas son peligros potenciales. La manipulación y desinfección inadecuadas de dichos instrumentos y suministros comprometería las medidas de control de infecciones dentro de la práctica de ortodoncia (Ayyed et al.; Carter; Grover et al.; Khatri et al.).

\section{Clasificación del instrumental}

Los instrumentos utilizados en ortodoncia pueden ser de tres categorías según el sistema de clasificación de Spaulding (1968). Este sistema divide el instrumental médico en categorias según el riesgo de infección relacionado con su uso. Esta ampliamente aceptado para determinar el grado de desin- 
fección o esterilización necesario para cada instrumental:

1. Crítico: Instrumental que entra en contacto con cavidades normalmente estériles dentro del organismo o tejido vascular. Penetran en los tejidos blando o duros de la cavidad oral. En el caso de ortodoncia corresponde al instrumental que penetra la mucosa. Ej: Bandas para molares, alicates para quitar bandas, kit de colocación de mini implantes, etc (Wichelhaus et al., 2006; Venturelli et al.; Jankare et al.; Marín, 2020; Kalra et al.)

2. Semicrítico: Instrumental que entra en contacto con la piel no intacta o mucosas. No penetran mucosas pero pueden estar en contacto con ellas o expuestas a saliva, sangre u otros fluidos. De preferencia deben estar estériles, en caso de no ser posible deben estar sometidos a desinfección de alto nivel. Ej: Alicates de ortodoncia, que por el gran número requerido por el paciente no es posible esterilizarlos entre paciente y paciente (Wichelhaus et al., 2006; Venturelli et al.; Ghanbarzadeh et al.; Marín; Kalra et al.; Jankare et al.).

3. No Critico: Instrumental que no entra en contacto con mucosas, solo toma contacto con piel sana, por lo que el riesgo de producir infecciones es mínimo. Solo necesitan desinfección de nivel intermedio entre pacientey paciente. Ej: Instrumentos o dispositivos que pueden entrar en contacto con aerosoles generados durante la atención dental como los controles de la unidad dental, mango de la lampara, lampara de fotocurado, base de la jeringa triple, pinzas de transferencia, mangueras de piezas de mano, etc (Wichelhaus et al., 2006; Venturelli et al.; Marín; Kalra et al; Jankare et al.).

\section{Clasificación de agentes desinfectantes}

De acuerdo con el tipo de agente patógeno que son capaz de destruir se clasifican en 3 niveles:

1.Nivel Alto: Eliminan todos los virus existentes y Mycobacterium tuberculosis. Deben posser amplio espectro, ser estables frente a materia orgánica, ser compatibles con el material de los equipos a desinfectar y poder medir su actividad o concentración por médio de indicadores químicos (Wichelhaus et al., 2006; Venturelli et al.; Marín).

- Glutaraldehído

- Formaldehído

- Ácido peracético
- Peróxido de Hidrógeno estabilizado

- Ortoftaldehído

2. Nivel Intermedio: Elimina formas vegetativas de virus, hongos y bacterias, pero no todos los virus de tamaño pequeño no lipídicos. En circunstancias especiales puede eliminar Mycobacterium tuberculosis (Wichelhaus et al., 2006; Venturelli et al.; Marín).

- Alcohol etílico $70 \%$

- Alcohol isopropílico 70 \% a $90 \%$

- Fenoles

- Asociaciones de aldehídos.

3. Nivel Bajo: Elimina bacterias patógenes en su forma vegetativa y algunos hongos. No elimina Mycobacterim tuberculosis ni virus de tamaño pequeño no lipídicos (Wichelhaus et al., 2006; Venturelli et al.; Marín).

- Hipoclorito de sodio a mil ppm.

- COMPUESTOS DE AMONIO CUATERNARIO.

\section{DISCUSIÓN}

La esterilización se puede realizar de varias formas. Algunas de las formas más comunes que se siguen en la práctica de la ortodoncia incluyen la esterilización en autoclave de vapor, la esterilización con calor seco, la esterilización con vapor químico y la esterilización con óxido de etileno. Sin embargo, dado al uso frecuente de los alicates de ortodoncia entre pacientes, no es posible someterlos a un proceso de esterilización constante. Al clasificarse como material semicrítico, es posible utilizar desinfección por métodos químicos (esterilización en frío). Este proceso consiste en poner en contacto la superficie del material con agentes químicos. Para lograr la desinfección de alto nivel hay que mantener el instrumental en inmersión por un tiempo determinado (Ghanbarzadeh et al.; Carvalho et al., 2015; Jain \& Patel; Marín; Turkistani). La selección de una solución desinfectante específica depende de una serie de factores tales como: toxicidad, corrosividad, tinción, precio, disponibilidad, nivel $y$ velocidad de la actividad antimicrobiana (Ghanbarzadeh et al.).

Los más frecuentes son el alcohol isopropílico al $70 \%$ y el glutaraldehído. Sin embargo, de acuerdo con el contexto de la pandemía por SARs Cov-2 y según la revisión bibliográfica actual, han sugerido otras alternativas para la limpieza del instrumental de ortodoncia. 
- Alcoholes: Componentes químicos solubles en agua. Los más utilizados son el alcohol etílico y el alcohol isopropílico. Destruyen formas vegetativas de bacterias, virus y hongos. Actúan precipitando ácidos nucleicos, desnaturalizando proteínas y disolviendo grasas, ejerciendo así su acción antimicrobiana. La concentración bactericida óptima fluctúa entre el 60 $\%$ al $90 \%$ por volumen, generalmente se utilizan al 70 $\%$. Se considera un desinfectante de nivel medio y se utiliza como desinfectante de superficies e instrumentos no críticos (Cabrera-Tasayco et al.; Venturelli et al.; Gurzawska-Comis et al.; Khatri et al.; Marín; Kalra et al.).

- Cloro y compuestos derivados: Tienen amplio espectro bactericida, sin embargo, su uso es limitado debido a que se inactivan en presencia de materia orgánica, son inestables y corroen el material metálico. Son tóxicos en contacto con piel y mucosas. El más utilizado corresponde al hipoclorito de sodio. Se utiliza para limpiar superficies e instrumental no crítico (CabreraTasayco et al.; Marín).

- Fenoles: En altas concentraciones actúa como tóxico del protoplasma penetrando y destruyendo la pared celular y precipitando las proteínas celulares. La evaluación de su eficacia ha tenido resultados contradictorios y se ha visto que es absorbido por el material poroso dejando residuos que no pueden ser eliminados. Se utiliza para la limpieza de superficies y de instrumental no crítico (Marín).

- Glutaraldehído: Es eficaz contra una gran variedad de microorganismos. Se utiliza en una concentración del $2 \%$ como un desinfectante de alto nivel. El mecanismo de acción se debe a la alquilación de los grupos amino, sulfidrilo, hidroxilo y carboxilo, los cuales alteran el ARN, ADN y la síntesis proteica en los microorganismos. Es activo en presencia de materia orgánica y puede actuar tanto como desinfectante (30 minutos) y como esterilizante (10 horas). Tiene como desventaja el nivel de toxicidad, que puede causar irritación, ulceración y quemaduras de la piel y las mucosas; también puede causar irritación a los ojos y al sistema respiratorio. Aparte de los problemas de toxicidad, la solución a base de aldehídos causa corrosión y oxidación de los instrumentos de acero inoxidable que comprenden la mayoría de los instrumentos dentales y de ortodoncia (Cabrera-Tasayco et al.; Carvalho et al.; Khatri et al.; Ministerio de Salud, 2018; Turkistani; Marín; Malekshoar et al., 2021).

- Ácido peracético: Su concentración de uso es al 606
$0,25 \%$. Es esporicida a bajas concentraciones y en contacto con materia orgánica; realiza la desinfección en 10 minutos y la esterilización entre 30 minutos y 1 hora. Su actividad microbicida se debe a la desnaturalización de proteínas, alteración de la permeabilidad de la pared celular y oxidación de puentes sulfildrilos y sulfuros en proteínas y enzimas. Además, es biodegradable, no tóxico y no corrosivo. Sin embargo, posee alta inestabilidad y alto costo (Cabrera-Tasayco et al.; Carvalho et al.; Ministerio de Salud; Turkistani; Marín; Malekshoar et al.).

- Ortoftaldehído: Aprobado su uso por la FDA. Su concentración de uso es de 0,55\%, aunque también se considera activo en una concentración del 0,3\% a una temperatura de $20^{\circ} \mathrm{C}$. Se necesita un tiempo de inmersión mínimo de 5 minutos. El período de reutilización de la solución no debe ser superior a 14 días, monitoreando su concentración (Marín; Ministerio de Salud).

De acuerdo con diferentes estudios se determino que el glutaraldehído al $2 \%$ puede descontaminar los alicates, se recomienda inmersión en una solución de glutaraldehído al $2 \%$ durante 30 minutos y luego enjuague con agua. Además, la utilización de las sustancias químicas glutaraldehído al $2 \%$ y el ácido peracético al $0,25 \%$ se vio que eliminan completamente los microorganismos, sin embargo, el alcohol isopropílico al $70 \%$ no pudo eliminar completamente S. aureus (Almeida et al.; CabreraTasayco et al.; Carvalho et al.; Omidkhoda et al., 2016; Khatri et al.).

En el caso del uso de ortoftaldehído se recomienda lavar el instrumental con agua y detergente enzimático, luego secar bien todas las partes del instrumental antes de sumergirlo en la solución desinfectante. Preocuparse que el instrumental quede completamente sumergido y dejar en un contenedor con tapa durante 10 minutos, como mínimo, a una temperatura de $20^{\circ} \mathrm{C}$ o mayor. Aclarar por inmersión con agua (solución esteril) como mínimo 1 minuto, hasta 3 veces. Proceder al secado (Marín; Ministerio de Salud).

El etanol $95 \%$ y el hipoclorito de sodio en concentración superior al 0,1\% son capaz de inactivar el coronavirus, por lo que se recomienda la aplicación de etanol $95 \%$ al instrumental inmediatamente después de la atención de ortodoncia y luego la desinfección de alto nivel. No así el uso de hipoclorito de sodio, ya que daña el metal de los alicates (Cabrera- 
Tasayco et al.; Gurzawska-Comis et al.; Marín; Malekshoar et al.).

El problema más común que puede encontrar un ortodoncista es el número limitado de juegos de alicates para tratar a los pacientes. La solución ideal es comprar juegos de alicates adecuados que supondrán una carga económica para el ortodoncista, o tratar a un menor número de pacientes cada día, lo que reducirá la productividad. Por lo tanto, una solución práctica consiste en dividir los instrumentos en alicates de uso regular (alicates de pico de pájaro, cortador de alambre de extremo distal, portaagujas de Mathew etc.), instrumentos de unión (pinzas de soporte, y calibre posicionador de soportes), y instrumentos de desunión (alicates de desunión y fresas de carburo de tungsteno). Esto ayudaría a prevenir la suciedad innecesaria de los instrumentos no utilizados permitiendo utilizarlos con otros pacientes. Otra forma de abordar este problema es mantener siempre un mínimo de tres juegos de alicates. Las citas deben programarse de tal manera que las citas de corta duración estén en secuencia seguidas de citas de duración intermedia y larga, lo que proporcionaría tiempo suficiente para esterilizar en autoclave los instrumentos usados ??para su uso posterior. Se debe seguir la regla de 321, lo que significa tener tres conjuntos de instrumentos y programar dos citas cortas seguidas de una cita larga / intermedia (Jain \& Patel).

Control de corrosión, lubricación y consideraciones especiales de los alicates de ortodoncia

El proceso de limpieza y esterilización de los instrumentos de ortodoncia presenta problemas ya que estos instrumentos tienen grandes áreas de bisagra que son difíciles de limpiar y esterilizar. También tienen márgenes cortantes, ángulos afilados y extremos puntiagudos que se dañan fácilmente. La corrosión de los alicates es una de las consecuencias de la esterilización que enfrentan los ortodoncistas (Wichelhaus et al., 2004; Khatri et al.; Jankare et al.). La corrosión correspone a un evento electroquímico que sufren los metales cuando reaccionan con un oxidante como resultado de reacciones de oxidación y reducción. En el contacto de dos metales diferentes 0 áreas diferentes dentro de un solo metal se establece una diferencia de potencial que resulta en un flujo de electrones. El flujo de electrones deja iones reactivos que se combinan fácilmente con el oxígeno atmosférico para formar óxidos. Condiciones como temperaturas extremas, abrasión física, galvanismo o iones reactivos que rompen la capa de óxido de cro- mo harán que el acero sea vulnerable a la corrosión (Jankare et al.).

Los protocolos de esterilización empleados para los alicates de ortodoncia afectan las propiedades físicas y mecánicas y poseen un alto riesgo de corrosión. El autoclave afecta negativamente a los instrumentos de ortodoncia, provocando el desafilado y la corrosión de sus filos cortantes. Sin embargo, estudios recientes no mostraron diferencias significativas en el desgaste medio, ya sea esterilizado con autoclave de vapor o calor seco. La corrosión cualitativamente más destructiva se asocia con la esterilización en frío, la esterilización por calor causa menos daño que la esterilización química. Sin embargo, ambas dañan instrumental de ortodoncia (Wichelhaus et al., 2004; Yezdani et al., 2015; Jankare et al.).

La corrosión también se puede prevenir engrasando las superficies de las juntas con las soluciones adecuadas.

La resistencia a la corrosión depende del manejo de los alicates y pinzas por parte del personal encargado del procedimiento. Siempre se recomienda seguir las indicaciones del fabricante para limpiar este tipo de instrumental (Wichelhaus et al., 2004).

\section{Para reducir la corrosión:}

- Limpiar y eliminar la suciedad de los instrumentos y enjuagar con agua destilada (Kalra et al.).

- El agua debe estar desionizada y ser de buena calidad (Kalra et al.).

- Secar con aire a presión antes de la esterilización. Si no se secan bien, la reacción iónica creará una capa suelta de óxido (Khatri et al.).

- Se aconseja la esterilización de los alicates preferiblemente en una posición abierta (García-Camba et al.).

- En el caso de la utilización de productos de desinfección químicos, estos deben ser aplicados siguiendo estrictamente las indicaciones del fabricante, y respetando la concentración correcta (Kalra et al.). - Los instrumentos cromados y los de acero inoxidable deben esterilizarse por separado porque la acción del electrolito puede transportar partículas de carbono del metal expuesto de un instrumento cromado y depositarse sobre el acero inoxidable (Kalra et al.).

- En caso de limpieza manual, evitar trabajar con objetos duros (raspador, cepillo metálico) ya que pueden destruir la superficie de los instrumentos incrementándose el riesgo de oxidación (Kalra et al.). 
- Es mejor mantener los instrumentos envueltos. Deben evitarse los detergentes con bases de cloruro porque los residuos de cloruro se unen con el vapor para formar hipoclorito ( $\mathrm{HCl}$ ) (Kalra et al.).

- Los detergentes con $\mathrm{pH}$ superior a 8,5 pueden alterar la capa de óxido de cromo (Kalra et al.).

- Si se esterilizan alicates o instrumentos oxidados junto a instrumentos intactos puede producirse la formación de óxido fino y la consiguiente destrucción del instrumento en buen estado (Khatri et al.; Kalra et al.).

- Los alicates e instrumentos no deben permanecer durante un tiempo prolongado en un medio húmedo (Khatri et al.).

\section{CONCLUSIONES}

El control eficaz de las infecciones debe ser un componente rutinario dentro de la práctica clínica. Para poder prevenir la posible propagación de enfermedades infecciosas, es importante que los profesionales dentales cuenten con información actualizada que pueda utilizarse para desarrollar un protocolo óptimo de asepsia.

El ortodoncista puede minimizar e incluso prevenir la posibilidad de una infección cruzada, siendo responsabilidad de cada ortodoncista realizar su práctica de una manera segura. Desde el surgimiento de la pandemia de COVID-19, el tratamiento de ortodoncia ha enfrentado muchos desafíos debido a su naturaleza a largo plazo. No existen estudios específicos que puedan aportar un apoyo real a la conducta clínica, ni protocolos definitivos respaldados por evidencia sólida para el desarrollo de la ortodoncia durante la pandemia, por lo que se requieren más estudios que sean de ayuda en la práctica futura.

MUNIZAGA, N. M. S.; ALVAREZ, P. E. A.; HEMPEL, S. G.; SAT, Y. M. I.; CARRANZA, M. F. \& TERÁN, Q. K. Disinfection of orthodontic pliers. recommendations update in the context of COVID-19. literature review. Int. J. Odontostomat., 15(3):602-609, 2021

ABSTRACT: Adequate knowledge of definitions, norms, procedures, use of antiseptics and disinfectants, allows to avoid the spread of infectious agents and provides knowledge for proper use of these aides. The objective of this study is to present the information available regarding the disinfection of orthodontic pliers in a structured and organized manner, and to provide a guide for optimal infection control. A search was carried out using PubMed, MEDLINE and Google Scholar, regardless of language, from the year 2000 to April 2021. The most recommended disinfectants for the disinfection of orthodontic pliers and the considerations in their use were established. In addition, risks of corrosion and recommendations to avoid them were determined. It is important to have up-to-date information that can be used to develop an optimal asepsis program, especially in the present context of a pandemic.

KEY WORDS: COVID, COVID-19, COVID-2019, 2019-nCoV, SARS-CoV-2, coronavirus, disinfection pliers, orthodontic pliers, disinfection, orthodontic pliers.

\section{REFERENCIAS BIBLIOGRÁFICAS}

Almeida, C. M. F.; Carvalho, A. S. \& Duarte, D. A. Evaluation of disinfection methods of orthodontic pliers. Dental Press J. Orthod., 17(4):105-9, 2012

Ayyed, A. B. Dental practice infection control measurements: Coronavirus Disease (COVID-19) Outbreaks. Int. J. Clin. Pediatr. Dent., 13(3):279-83, 2020.

Cabrera-Tasayco, F. D. P.; Rivera-Carhuavilca, J. M.; Atoche-Socola, K. J.; Peña-Soto, C. \& Arriola-Guillén, L. E. Biosafety measures at the dental office after the appearance of COVID-19: a systematic review. Disaster Med. Public Health Prep., 1-5, 2020.

Carter, A. Can orthodontic care be safely delivered during the COVID19 pandemic? Recommendations from a literature review. Evid. Based Dent., 21(2):66-7, 2020.

Carvalho, M. R.; dos Santos da Silva, M. A.; de Sousa Brito, C. A.; Campelo, V.; Kuga, M. C.; Tonetto, M. R.; De Jesus Tavarez, R. R.; Bandéca, M. C. \& Pinzan-Vercelino, C. R. Comparison of antimicrobial activity between chemical disinfectants on contaminated orthodontic pliers. J. Contemp. Dent. Pract., 16(8):619-23, 2015.

Çelikel, A. D. G.; Ekmekçioglu, H.; Külekçi, G. \& Fıratı, S. Evaluation of the compliance of orthodontists to infection control procedures in Turkey. Turk. J. Orthod., 31(2):37-49, 2018.

García-Camba, P.; Marcianes, M. \& Varela Morales, M. Changes in orthodontics during the COVID-19 pandemic that have come to stay. Am. J. Orthod. Dentofacial Orthop., 158(4):e1-e3, 2020.

Ghanbarzadeh, M.; Mahboobe, D.; Ghazvini, K. \& Movahhed, T. Disinfection of orthodontic pliers using three different disinfectants. J. Int. Dent. Med. Res., 1-6, 2014.

Grover, I.; Agrawal, A.; Kaur, H.; Soni, R.; Mihani, L. \& Grover, M. Managing airborne isolation and precautions in orthodontic practice during the outbreak of coronavirus disease 2019: An orthodontist perspective. Saint Int. Dent. J., 4:11-6, 2020.

Guo, Y.; Jing, Y.; Wang, Y.; To, A.; Du, S.; Wang, L. \& Bai, D. Control of SARS-CoV-2 transmission in orthodontic practice. Am. J. Orthod. Dentofacial Orthop., 158(3):321-9, 2020.

Gurzawska-Comis, K.; Becker, K.; Brunello, G.; Gurzawska, A. \& Schwarz, F. Recommendations for Dental Care during COVID19 Pandemic. J. Clin. Med., 9(6):1833, 2020.

Jain, M. \& Patel, D. Overcoming the Challenges of COVID-19 pandemic in orthodontic practice. Front. Dent., 17:12, 2020.

Jankare S.; Surani, S. S.; Parchake, P.; Borkar, E. \& Rathod, A. Sterilization protocol in orthodontic practice: a review. Acta Sci. Dent. Sci., 3(12):32-9, 2019.

Kalra, S.; Tripathi, T. \& Rai, P. Infection control in orthodontics. J. Orthod. Endod., 1(1):1, 2015.

Kaur, H.; Kochhar, A. S.; Gupta, H.; Singh, G. \& Kubavat, A. Appropriate orthodontic appliances during the COVID-19 pandemic: A scoping review. J. Oral Biol. Craniofac. Res., 10(4):782-7, 2020. 
MUNIZAGA, N. M. S.; ALVAREZ, P. E. A.; HEMPEL, S. G.; SAT, Y. M. I.; CARRANZA, M. F. \& TERÁN, Q. K. Desinfección de alicates de ortodoncia. Actualización de recomendaciones en contexto de COVID- 19. Revisión de la Literatura. Int. J. Odontostomat., 15(3):602-609, 2021.

Khatri, J. M.; Jadhav, M. M. \& Tated, G. H. Sterilization and orthodontics: A literature review. Int. J. Orthod. Rehabil., 8:1416, 2017.

Kondody, R. T.; Sana, S.; Reddy, R.; Fatima, A. \& Bangi, S. L. Coronavirus disease 2019. Challenges today and tomorrow in orthodontic practice: a review. Turk. J. Orthod., 34(1):61-7, 2021.

Malekshoar, M.; Malekshoar, M. \& Javanshir, B. Challenges, limitations, and solutions for orthodontists during the coronavirus pandemic: A review. Am. J. Orthod. Dentofacial Orthop., 159(1):e59-e71, 2021.

Marín, R. M. Desinfección de Alicates de Ortodoncia. Hospital Luis Calvo Mackenna. Recomendaciones en Contexto de COVID19. Mayo 2020. Santiago de Chile, Hospital Luis Calvo Mackenna, 2020. Disponible en: https://webdental.cl/odontologia/ wp-content/uploads/2020/06/DesinfeccionAlicatesOrtodonciaCoVid19.pdf

Ministerio de Salud. Norma General Técnica N¹99 sobre Esterilización y Desinfección de Alto Nivel y Uso de Artículos Médicos Estériles en Establecimientos de Atención en Salud. Santiago de Chile, Ministerio de Salud, Gobierno de Chile, 2018. Disponible en: https://www.minsal.cl/wp-content/uploads/2018/03/Norma-General-T \%C3 \%A9cnica-N \%C2 \%B0-199-sobreesterilizaci \%C3 \%B3n-y-desinfecci \%C3 \%B3n-de-alto-nivel-yuso-de-art \%C3 \%ADculo-m \%C3 \%A9dicos-est \%C3 $\%$ A9riles.pdf

Omidkhoda, M.; Rashed, R.; Bagheri, Z.; Ghazvini, K. \& Shafaee, $\mathrm{H}$. Comparison of three different sterilization and disinfection methods on orthodontic markers. J. Orthod. Sci., 5:14-7, 2016.

Spaulding, E. H. Chemical Disinfection of Medical and Surgical Materials. En: Lawrence, C. \& Block, S. S. Disinfection, Sterilization, and Preservation. Philadelphia, Lea \& Febiger, 1968. pp.517-31.

Suri, S.; Vandersluis, Y. R.; Kochhar, A. S.; Bhasin, R. \& Abdallah, M. N. Clinical orthodontic management during the COVID-19 pandemic. Angle Orthod., 90(4):473-84, 2020.

Turkistani, K. A. Precautions and recommendations for orthodontic settings during the COVID-19 outbreak: A review. Am. J. Orthod. Dentofacial Orthop., 158(2):175-81, 2020.

Venturelli, A. C.; Torres, F. C.; Almeida-Pedrin, R. R.; Rodrigues de Almeida, R.; de Almeida M. R. \& Carvalho Ferreira, F. P. Avaliação microbiológica da contaminação residual em diferentes tipos de alicates ortodônticos após desinfecção com álcool 70 \%. Rev. Dent. Press Ortodon. Ortop. Facial, 14(4):43-52, 2009.

Wichelhaus, A.; Bader, F.; Sander, F. G.; Krieger, D. \& Mertens, T. Effective disinfection of orthodontic pliers. J. Orofac. Orthop., 67(5):316-36, 2006.

Wichelhaus, A.; Brauchle, G.; Mertmann, M. \& Sander, F. G. Corrosion of orthodontic pliers using different sterilization procedures. J. Orofac. Orthop., 65(6):501-11, 2004.

Yezdani, A.; Mahalakshmi, K. \& Padmavathy, K. Orthodontic instrument sterilization with microwave irradiation. J. Pharm. Bioallied Sci., 7(Suppl. 1):S111-5, 2015.

\author{
Dirección para correspondencia: \\ María Soledad Munizaga Naveillan \\ Facultad de Odontología \\ Universidad de los Andes \\ Santiago \\ CHILE
}

E-mail: msmunizaga@miuandes.cl 